\title{
Genetic Diversity and New Lineages of Dengue Virus Serotypes 3 and 4 in Returning Travelers, Germany, 2006-2015
}

\author{
Sami Shihada, Petra Emmerich, \\ Corinna Thomé-Bolduan, Stephanie Jansen, \\ Stephan Günther, Christina Frank, \\ Jonas Schmidt-Chanasit, Daniel Cadar
}

During 2006-2015, we analyzed 70 dengue virus (DENV) strains isolated from febrile travelers returning to Germany. High genetic diversity, including multiple co-circulating DENV lineages and emerging new lineages of DENV-3 and DENV-4, was demonstrated. Our passive surveillance system based on returning travelers yielded substantial information on DENV diversity.

A lthough dengue virus (DENV) infects $\approx 390$ million persons annually and one third of the world's population is at risk for infection, there is no effective vaccine or specific antiviral therapy for infection with DENV (1). Dengue is a rapidly spreading mosquito-borne viral disease and the most frequent cause of febrile illness among international travelers returning from DENV-endemic tropical areas, such as Southeast Asia, the western Pacific region, and the Americas $(2,3)$. Viremic travelers have the potential to introduce DENV into DENV-free or nonendemic areas where competent mosquito vectors are present (4). Reintroduction of DENV in regions that had been considered free of the disease for many years has also been observed (5-7).

Phylogenetic analysis has elucidated the origins, epidemiology, and forces that shape DENV molecular evolution in nature (8). For example, according to official German air travel statistics reports, 4,855,763 air trips were taken in 2011 from Germany to countries listed as DENV-endemic areas by the World Health Organization; $10 \%-20 \%$ each of these trips were made to India, Thailand, and Brazil; and $5 \%-10 \%$ each flew to Singapore, Mexico, and the Dominican Republic (9). We determined the genetic relatedness and molecular epidemiology of DENV isolates from travelers returning to Germany during 2006-2015.

\footnotetext{
Author affiliations: Bernhard Nocht Institute for Tropical

Medicine, Hamburg, Germany (S. Shihada, P. Emmerich,

C. Thomé-Bolduan, S. Jansen, S. Günther, J. Schmidt-Chanasit,

D. Cadar); Robert Koch Institute, Berlin, Germany (C. Frank);

German Centre for Infection Research, Hamburg

(J. Schmidt-Chanasit)
}

DOI: http://dx.doi.org/10.3201/eid2302.160751

\section{The Study}

During 2006-2015, we analyzed 15,876 acute-phase serum samples from patients with suspected DENV infection; the samples had been submitted to the World Health Organization Collaborating Centre for Arbovirus and Hemorrhagic Fever Reference and Research for diagnostic testing. We tested all samples by using DENV type-specific real-time reverse transcription PCR (rRT-PCR) (10) or an antigencapture ELISA (Platelia Dengue NS1 Ag; Bio-Rad, Hercules, CA, USA) and in-house DENV IgG and IgM indirect immunofluorescence assays. rRT-PCR- and nonstructural protein 1-positive serum samples that tested negative for DENV IgG and IgM were spread onto Vero E6 cells and incubated for 7 days at $37^{\circ} \mathrm{C}$; successful DENV isolation was identified by rRT-PCR. We extracted viral RNA from cell culture supernatants by using the QIAamp Viral RNA Mini Kit (QIAGEN, Hilden, Germany).

We successfully isolated $70 \mathrm{DENV}$ strains originating from 20 countries (online Technical Appendix, http://wwwnc.cdc.gov/EID/article/23/2/16-0751-Techapp1.pdf). We amplified the complete envelope glycoprotein (E) gene using DENV type-specific degenerate primers (online Technical Appendix). Sequence assembly, analysis, and multiple alignments were performed with Geneious version 7.1.8 (Biomatters, Auckland, New Zealand). All available complete envelope gene sequences of DENV serotypes 1-4 (DENV-1-4), except laboratory strains and potential recombinants, were retrieved from GenBank and compared with those sequenced in this study. The phylogenetic relationships and origin of the imported DENV isolates were analyzed by the maximum likelihood method in the RAxML program (11) with general time-reversible plus gamma distribution substitution model and a rapid bootstrap (100 replicates) procedure, and visualized in FigTree version 1.4.3 (http://tree.bio.ed.ac.uk/software/figtree/).

Most DENV infections were acquired in Thailand (35.7\%), followed by Indonesia (12.8\%), the Philippines $(10 \%)$, and India (7.1\%). The proportion of cases imported from other countries in Asia, Africa, and the Americas ranged between $1.4 \%$ and $4.3 \%$ (Figures 1,2 ). Phylogenetic analysis revealed a high genetic diversity of DENV1-4 in travelers from Germany, including co-circulation of multiple genetically diverse viral lineages that were closely related to those previously circulating in the Americas and Southeast Asia (mostly Thailand, Indonesia, and 


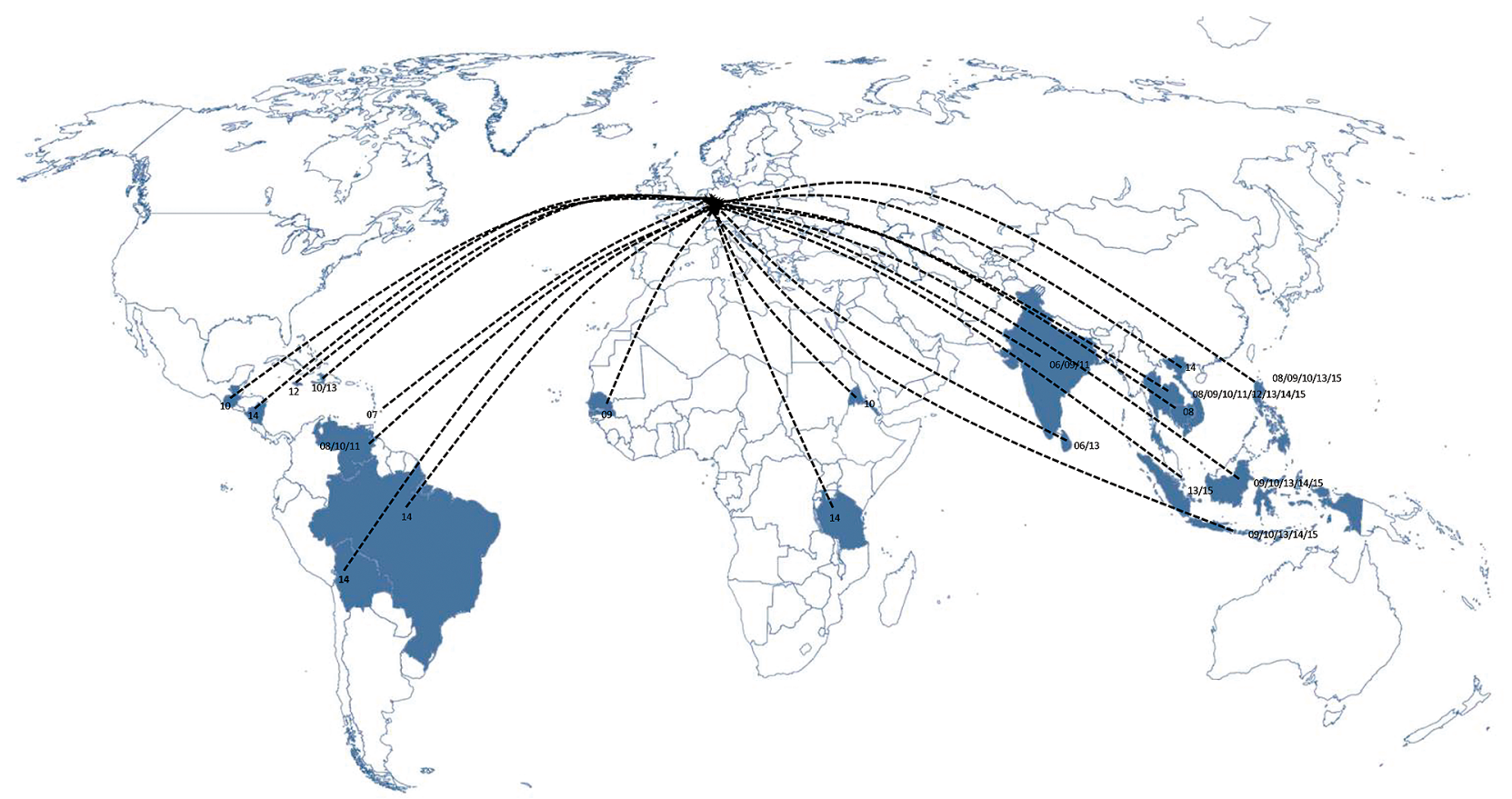

Figure 1. Geographic origin of dengue viruses isolated from travelers returning to Germany, 2006-2015.

the Philippines) (online Technical Appendix). DENV-1 was the predominant and most genetically diverse type, representing 33 sequences and clustering into 23 phylogenetically distinct lineages. Most of the isolates belonged to genotypes I and II, which are circulating in Southeast Asia (12). These genotypes represent dominant regional variants; only a few strains are closely related to viruses circulating in the Americas (genotype $\mathrm{V}$, lineages 3-6) or to a genotype III virus in India. All but 3 DENV-2 isolates were the Cosmopolitan genotype, and the source population was limited mostly to countries in Southeast Asia (online Technical Appendix).

DENV-2 phylogeny provides evidence for 7 phylogenetically distinct introductions of the virus into travelers from Germany; the origins of these isolates were predominantly in Bali and the Philippines. DENV-3 sequences belonged to genotypes I-III; these isolates were closely related to lineages representing regional or local variants, except lineages 8 and 9 (online Technical Appendix).

A key phylogenetic pattern was the presence of a new lineage, L6 (online Technical Appendix), within DENV3 phylogeny comprising closely related isolates sampled from German, Taiwanese, and Chinese travelers returning from Thailand and Laos during 2012-2014. A similar pattern of clustering to that for DENV-3 was observed for DENV-4 isolates. Thus, most DENV-4 from travelers were infected with genotype I and III strains closely related to lineages commonly sampled within Southeast Asia and to a genotype II isolate from Bolivia. A key phylogenetic pattern was the presence of a new lineage, L3, within DENV-4 phylogeny (online Technical Appendix) with sequences found in samples from patients tested during 2013-2015 with travel histories to Thailand, China, and the Philippines (lineage 3). Lineage 3 was the most recently circulating lineage detected in this study. This clustering is compatible with extensive viral traffic between Thailand, China, and the Philippines; Thailand, where most of the DENV infections were acquired, is thus a possible source of a virus population responsible for local or regional outbreaks.

\section{Conclusions}

Countries in Southeast Asia that are considered DENV hyperendemic are increasingly popular tourist destinations for residents of Germany; thus, German travelers to these countries are potentially exposed to multiple types and genotypes of DENV. A high prevalence of DENV infection has been reported in travelers returning from DENV-endemic areas, emphasizing the importance of international travelers as potential sources of imported disease or sentinels for local outbreaks in DENV-free or non-DENV-endemic areas $(2,3)$. The relative risk of infection by country is difficult to calculate without attention to seasonal fluctuations in dengue fever incidence and travel patterns. Broadly speaking, though, among the top contributing countries mentioned, the comparative risk of infection with travel-associated DENV appears much higher in the Philippines and Indonesia (10\% and 12.8\% of the cases, compared with $1.7 \%$ and $2.8 \%$, respectively, of travelers from Germany to DENV-endemic countries) than in Thailand $(35.7 \%$ of the cases versus $15.0 \%$ of the 
travelers) and the lowest in India (7.1\% of the cases versus $18.5 \%$ of the travelers).

We investigated DENV diversity and origin of infection in travelers returning to Germany from DENVendemic areas and identified a high genetic diversity of DENV genotypes and lineages. Notably, 2 of these lineages (DENV-3, genotype III, lineage 6 and DENV-4, genotype III, lineage 6) appear to have emerged very recently and are still responsible for local outbreaks in countries in Southeast Asia, thus reiterating the need to monitor the appearance and spread of novel lineages. Most investigated isolates were closely related to lineages known to have circulated in Thailand, the Philippines, and Indonesia, indicating that these countries serve
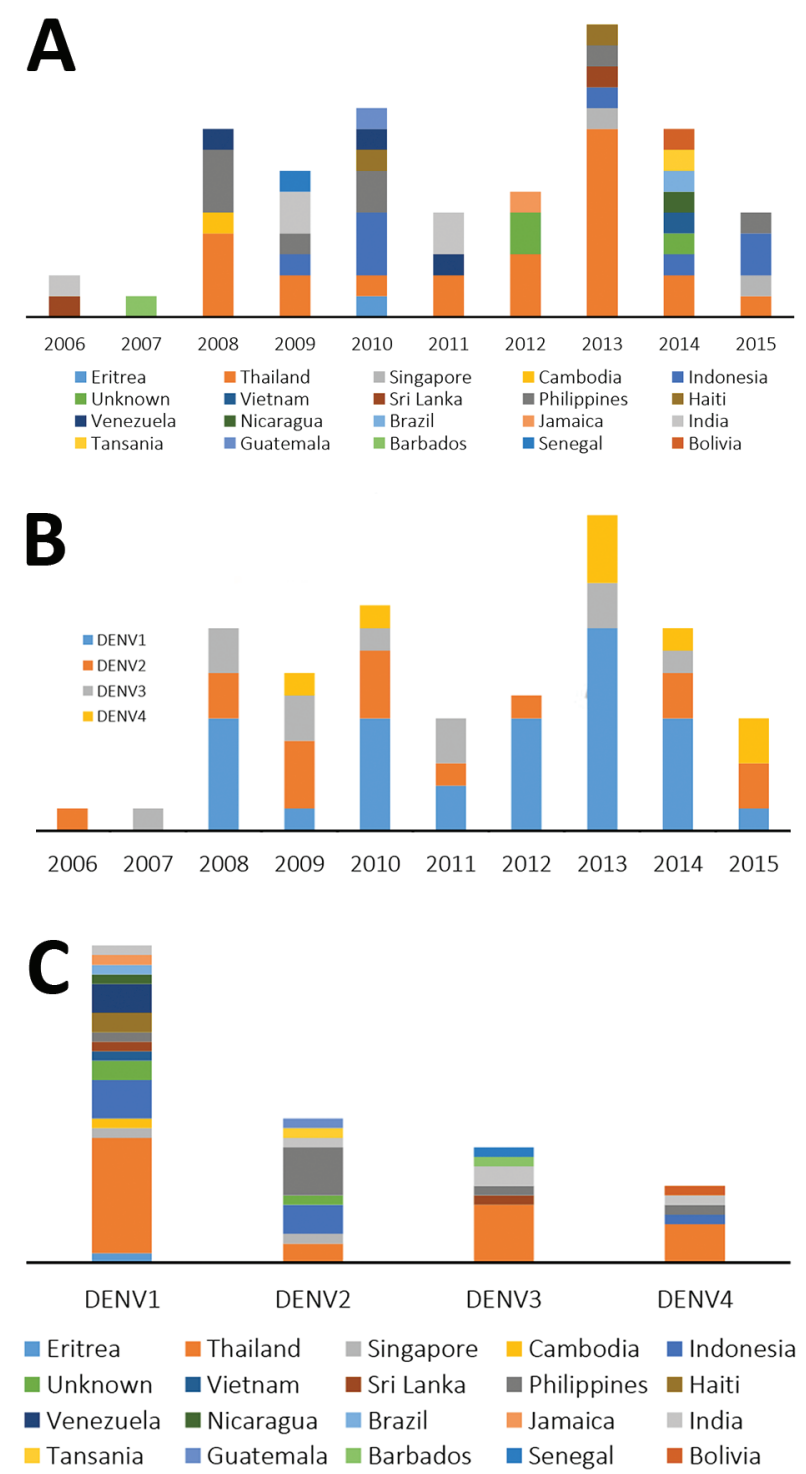

Figure 2. Year of introduction $(A)$ and diversity (B and $C$ ) of dengue viruses isolated from travelers returning to Germany, 2006-2015. as a major source of multiple DENV lineages (12). The observed high numbers of co-circulating lineages in the Thai, Indonesian, and Philippines source populations support the hypothesis of multiple geographic origins or extensive virus interchange among these countries (online Technical Appendix).

Surveillance of symptomatic returned travelers can provide information on circulating DENV genotypes and lineages in heavily visited tourist areas and DENV-endemic regions. In Europe, the emergence of arboviruses should be particularly monitored because of the introduction and expansion of the DENV vector Aedes albopictus mosquito. In Germany, where recently introduced Ae. albopictus mosquitos have spread in the southwestern part of the country, international travelers and the presence of competent vectors could potentially facilitate seasonal local transmission of DENV $(7,13,14)$. Our findings indicate a diverse array of imported DENV infections in travelers from Germany and emphasize the need for a continued surveillance of DENV infections in non-DENV-endemic regions as well as prompt and rapid serologic and molecular testing for DENV infection in febrile patients returning from DENV-endemic countries.

\section{Acknowledgment}

We thank Mathis Petersen for excellent technical assistance.

Dr. Shihada is a physician at the Bernhard Nocht Institute for Tropical Medicine in Hamburg, Germany. His research interest is characterization of human and zoonotic viruses.

\section{References}

1 Bhatt S, Gething PW, Brady OJ, Messina JP, Farlow AW, Moyes CL, et al. The global distribution and burden of dengue. Nature. 2013;496:504-7. http://dx.doi.org/10.1038/nature12060.

2. Jelinek T, Mühlberger N, Harms G, Corachán M, Grobusch MP, Knobloch J, et al.; European Network on Imported Infectious Disease Surveillance. Epidemiology and clinical features of imported dengue fever in Europe: sentinel surveillance data from TropNetEurop. Clin Infect Dis. 2002;35:1047-52. http://dx.doi.org/10.1086/342906

3 Wilder-Smith A, Tambyah PA. Severe dengue virus infection in travelers. J Infect Dis. 2007;195:1081-3. http://dx.doi.org/ $10.1086 / 512684$

4. Schwartz E, Weld LH, Wilder-Smith A, von Sonnenburg F, Keystone JS, Kain KC, et al.; GeoSentinel Surveillance Network. Seasonality, annual trends, and characteristics of dengue among ill returned travelers, 1997-2006. Emerg Infect Dis. 2008;14:1081-8. http://dx.doi.org/10.3201/eid1407.071412

5. La Ruche G. Souarès Y, Armengaud A, Peloux-Petiot F, Delaunay P, Desprès P, et al. First two autochthonous dengue virus infections in metropolitan France, September 2010. Euro Surveill. 2010;15:19676.

6. Gjenero-Margan I, Aleraj B, Krajcar D, Lesnikar V. Klobučar A, Pem-Novosel I, et al. Autochthonous dengue fever in Croatia, August-September 2010. Euro Surveill. 2011;16:pii: 19805.

7. Becker N, Geier M, Balczun C, Bradersen U, Huber K, Kiel E, et al. Repeated introduction of Aedes albopictus into Germany, 
July to October 2012. Parasitol Res. 2013;112:1787-90. http://dx.doi.org/10.1007/s00436-012-3230-1

8. Weaver SC, Vasilakis N. Molecular evolution of dengue viruses: contributions of phylogenetics to understanding the history and epidemiology of the preeminent arboviral disease. Infect Genet Evol. 2009;9:523-40 http://dx.doi.http://dx.doi.org/ 10.1016/j.meegid.2009.02.003

9. Federal Statistical Office(DESTATIS). Metadata of a publication. Air transport series 8 series 6 (monthly reports) [in German]. Wiesbaden, 2011/2012 [cited 2016 Dec 14]. https://www.destatis. de/DE/Publikationen/Thematisch/TransportVerkehr/Luftverkehr/ Luftverkehr.htm

10. Johnson BW, Russell BJ, Lanciotti RS. Serotype-specific detection of dengue viruses in a fourplex real-time reverse transcriptase PCR assay. J Clin Microbiol. 2005;43:4977-83. http://dx.doi.org/10.1128/JCM.43.10.4977-4983.2005

11. Stamatakis A, Hoover P, Rougemont J. A rapid bootstrap algorithm for the RAxML web servers. Syst Biol. 2008;57:758-71. http://dx.doi.org/10.1080/10635150802429642
12. Ernst T, McCarthy S, Chidlow G, Luang-Suarkia D, Holmes EC, Smith DW, et al. Emergence of a new lineage of dengue virus type 2 identified in travelers entering western Australia from Indonesia, 2010-2012. PLoS Negl Trop Dis. 2015;9:e0003442. http://dx.doi.org/10.1371/journal.pntd.0003442

13. Werner D, Kronefeld M, Schaffner F, Kampen H. Two invasive mosquito species, Aedes albopictus and Aedes japonicus japonicus, trapped in south-west Germany, July to August 2011. Euro Surveill. 2012;17: pii:20067.

14. Pluskota B, Jöst A, Augsten X, Stelzner L, Ferstl I, Becker N. Successful overwintering of Aedes albopictus in Germany. Parasitol Res. 2016;115:3245-7. http://dx.doi.org/10.1007/s00436-016-5078-2.

Address for correspondence: Jonas Schmidt-Chanasit, Bernhard Nocht, Institute for Tropical Medicine, World Health Organization Collaborating Centre for Arbovirus and Hemorrhagic Fever Reference and Research, Bernhard-Nocht-Strasse 74, 20359 Hamburg, Germany; email: jonassi@gmx.de

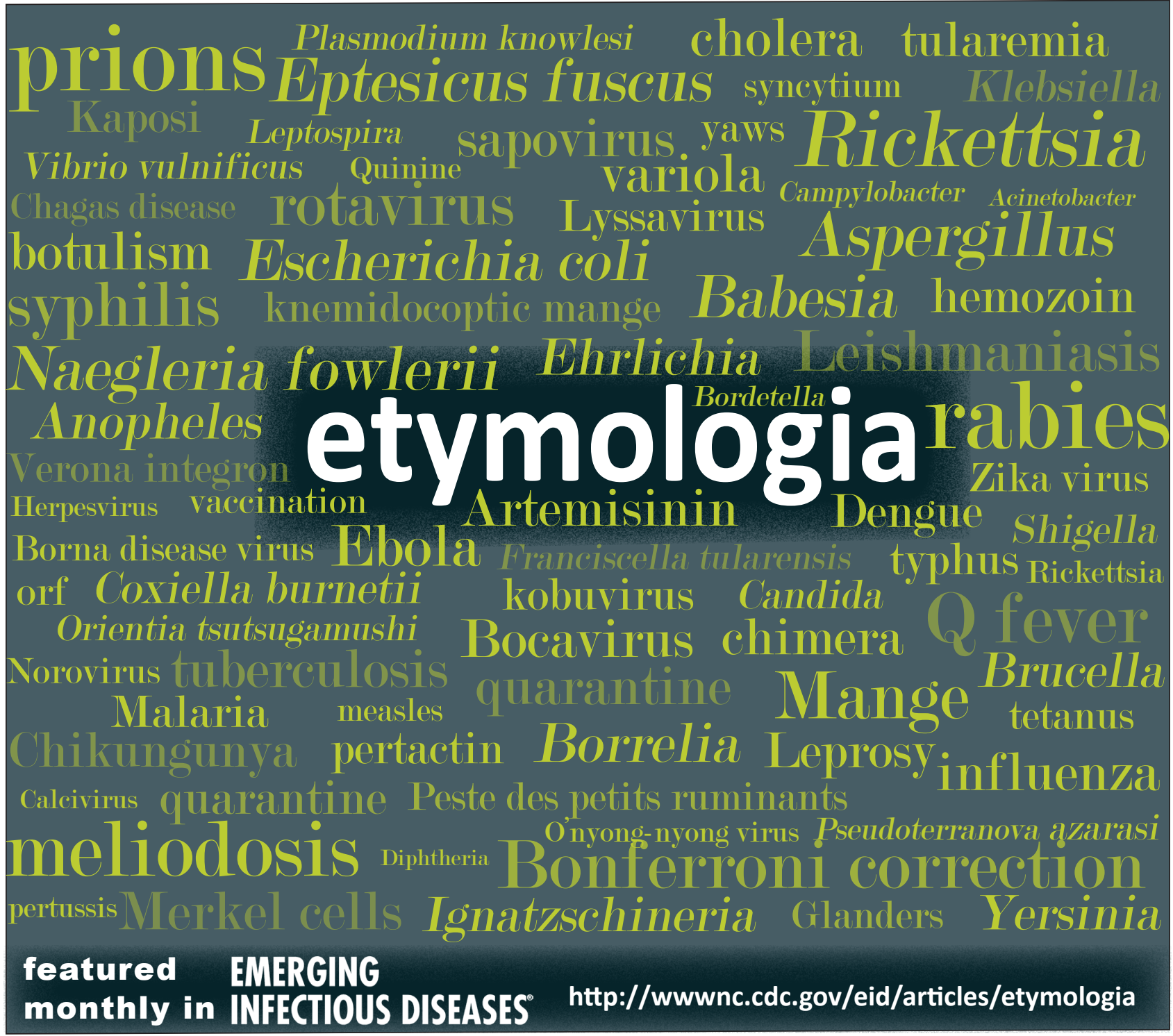

